\title{
中国移动 IP 城域网建设思路
}

\section{Construction Ideas of China Mobile IP MAN \\ 秦书瑞 胡富 路欢欢}

Shurui Qin $\mathrm{Fu} \mathrm{Hu}$ Huanhuan $\mathrm{Lu}$

广州杰赛科技股份有限公司

中国·广东 广州 510310

GCI Science \& Technology Co.,Ltd.,

Guangzhou, Guangdong, 510310, China
【摘 要】论文通过对 IP 城域网网络现状及业务需求的分析,提出中国移动的建设思路和 发展方向, 对 IP 城域网的建设有一定指导意义

(Abstract)Based on the analysis of IP MAN network status and business needs, this paper puts forward the construction ideas and development direction of China Mobile, which has a certain guiding significance for the construction of IP MAN.

【关键词】网络; 扁平化; 设计精细化; 可靠性

【Keywords】network; flatten; designing refinement; reliability

【DOI】10.36012/etr.vli4.711

\section{1 引言}

2019 年,政府工作报告中提出“持续推动网络提速 降费, 开展城市千兆宽带人户示范, 改造提升远程教 育、远程医疗网络, 推动移动网络扩容升级, 让用户切 实感受到网速更快更稳定。”另外, 从 2015 年开始, 传 统的大流量的下载类应用逐步萎缩, 而视频类应用快 速增长。随着用户规模增长和大带宽业务推动, 用户流 量会有爆发式的增长, 势必会对 IP 城域网承载能力提 出更高的要求。

\section{2 网络现状}

中国移动 IP 城域网介于 CMNet 省网与用户端设 备之间，提供多种业务在省内/城域内的互联及 CMNet 骨干网/省网的接人, 并且保证各种业务的安全性和服务质量 ${ }^{[1]}$ 。 经过多年的建设,已经形成了一个网络层次清晰、功能较为完 善的三层结构的全路由网络, 全网可支持 MPLS VPN、QOS 保 障、组播等技术。IP 城域网主要划分为核心层、业务控制层、 汇聚层。IP 城域网结构如图 1 所示。

(1)核心层: 由双节点核心路由器组成; 是 BRAS/SR 的接 人汇聚点, 是省网在地市的接入点, 负责城域内流量及与骨干 网之间的高速转发。

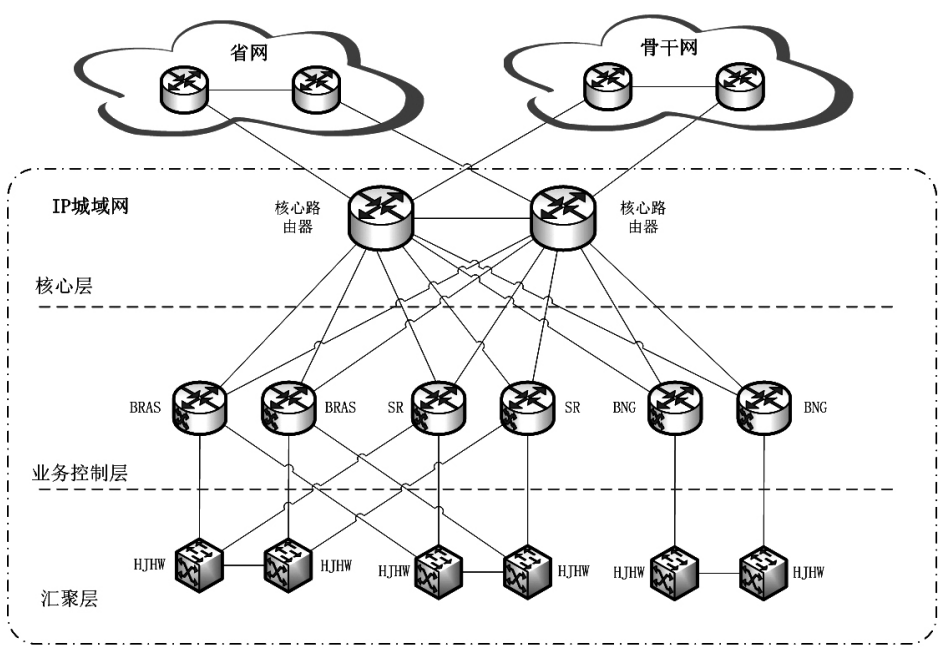

图 1 IP 城域网结构示意图

(2)业务控制层: 包括双边缘设备 (SR 和 BRAS) 和单边缘 设备 $(\mathrm{BNG})$ 。 BRAS/SR 分别为家客、集客业务提供网络接入, $\mathrm{BNG}$ 为 BRAS 和 $\mathrm{SR}$ 的合设设备。

(3)汇聚层: 由多台汇聚交换机组成,用于汇聚接入网各类 接人设备, 同时,将不同流量分流到不同控制设备终结。

\section{3 需求分析}

(1)网络结构需继续向扁平化演进,形成高并发、低时延的 网络架构。(2)进一步提升业务支持能力,提升端到端大带宽能 
实验与研究 Experiments and Research

力和网络性能, 提升组网灵活性和自动化配置能力。(3)加强业 务和流量精细化分析, 提升网络能力配置效率。带宽利用率不 均衡, 影响业务整体质量。(4)加强与传输、接入网络协同,端到 端提升网络可靠性。

\section{4 建设思路}

\section{1 推进网络扁平化和结构优化}

推进简洁、扁平网络架构, 满足千兆光宽、互联网电视、 $4 \mathrm{G}$ 上网等带宽及时延需求; 继续推进城域网双跨, 减轻省网 汇接层压力。

网络结构由三层结构向 “核心层+业务控制层” 结构演 进 ${ }^{[2]}$ 。逐步减少直至取消汇聚层, 减少业务跳数, 降低时延及 故障点。

双边缘结构向单边缘结构演进。现阶段，融合 BRAS 和 SR 功能的综合业务接入控制设备已成熟, 建议在业务控制层 采用 BRAS 和 SR 合设设备同时接入家宽和集团客户，以降 低设备圥余成本, 提高设备利用率。

\section{2 提升城域网设备能力及带宽能力}

持续推动大容量、高性能设备引人, 提升容量, 构建高速 通道, 快速疏导互联网流量和视频流量, 进一步提升设备级和 网络级的圥余保护能力。

(1)核心层:结合宽带提速、业务发展需求和主流厂家设备 演进路标, 建议核心层设备平台能力适度超前布局。大平台单 机相比小平台集群，在节省能耗和机房空间上更有优势, 因 此, CR 有新建或平台升级需求的,应以 $400 \mathrm{G} 、 800 \mathrm{G} / \mathrm{T}$ 级平台 为主;对于 $100 \mathrm{G}$ 平台等小容量设备不应继续扩容,避免投资 浪费。

(2)业务控制层:按需引入 $200 \mathrm{G}$ 平台和 $100 \mathrm{G}$ 上行链路。加 快小容量 BRAS 业务迁移至 BNG, 保障大带宽业务的真实接 人能力; 小容量 BRAS(40G 以下)不应继续扩容并逐步退网。

(3)汇聚层: 依据网络扁平化思路,汇聚层应控制并逐步减 小 HJSW 规模, 满足条件的 HJSW 退网。

(4)端到端链路设计要考虑 IP 优化, 建议直接利用光纤连 接，后续升级至更高带宽时只需将链路两端的设备端口升级 至更高容量,而不受传输系统建设的约束。

\section{3 城域网业务保障能力}

科学制定端到端网络利用率和性能指标, 保障业务体验。
端到端分析流量需求, 实现带宽合理配置; 按需进行 CDN POP 节点直连 BNG 和组播复制点下移, 减少业务跳数, 以降 低丢包可能性和网络时延。

提升网络智能化和灵活性, 满足政企上云等新业务需求。 统筹考虑业务运营管理要求, 提升 “云+网能力”端到端自动 化配置, 满足政企上云等新业务需求; 积极开展网络云化、软 件化技术探索,逐步推动网络重构。

\section{4 提升网络可靠性}

加强与传输协同规划, 实现设备端口、物理链路的主备, 完善流量经多路由负荷分担 ${ }^{[3]}$ 。BNG/BRAS/SR 至 CR 多个互 联端口应分布在不同槽位, 上行链路应保证至少两个以上物 理路由, 对于暂时不具备两个物理路由的, 应加强与传输专业 的衔接, 逐步完成双路由改造, 提升网络和业务安全性。

有效衔接 OLT 需求, 逐步提升 OLT-BNG/BRAS 间网络 可靠性。单个 OLT 承载超过 2000 户的, OLT 应采用双上联、 双路由方式实现链路保护; 推进双路由改造, 对现阶段不具备 双物理路由条件的, 应制定网络可靠性策略。

\subsection{IPv4 向 IPv6 转换}

目前, IP 城域网处于 IPv4 向 IPv6 转换的过程阶段。一方 面, IP 城域网内所有设备均需启用和配置 IPv6; 另一方面, 先 将一部分宽带用户改造为 IPv4/IPv6 双栈用户, 进而逐步推进 IPv4 的用户转化为 IPv6 用户的比例。

\section{5 结语}

从中远期来看, $5 \mathrm{G}$ 将在 $4 \mathrm{G}$ 不限量套餐实现快速流量增 长的基础上, 推动流量更快的增长; 物联网、虚拟现实(VR)和 增强现实 $(\mathrm{AR})$ 在未来将成为主流应用, 推动流量快速增长和 网络能力云化; SDN/NFV 技术及 BRAS 的应用将推动网络架 构的变革。

\section{参考文献}

[1]姜智峰.运营商互联网 IPv6 网络技术演进探讨 [J].移动通信, 2010(9):39-43.

[2]莫华国.基于多业务的 IP 城域网优化思路及方案 [J].电脑与电 信,2007(7):44-46.

[3]许化栋.4K 业务对 IP 城域网建设的影响[J].电信网技术,2015 (8):40-43. 